\title{
Influência do diâmetro e da fase folicular sobre a competência in vitro de oócitos obtidos de novilhas da raça Nelore
}

\author{
[Influence of diameter and follicular fase on the in vitro competence of oocytes from Nelore heifers] \\ C. Castilho ${ }^{1,3}$, G.S. Assis $^{2}$, J.M. Garcia ${ }^{2}$ \\ ${ }^{1}$ Aluna de pós-graduação - FCAV-UNESP - Jaboticabal, SP \\ ${ }^{2}$ Faculdade de Ciências Agrárias e Veterinárias \\ Via de Acesso Prof. Paulo Donato Castellane, s/n \\ 14884-900 - Jaboticabal, SP \\ ${ }^{3}$ Faculdade de Medicina Veterinária - UNOESTE - Presidente Prudente, SP
}

\begin{abstract}
RESUMO
Avaliou-se o efeito do diâmetro e da fase do desenvolvimento folicular sobre a competência de oócitos para a produção in vitro de embriões bovinos. A primeira onda folicular foi sincronizada com progestógeno por nove dias e 24 horas após a sua retirada aplicou-se LH. Os ovários foram recuperados 60h (G-60), 96h (G-96) e 108h (G-108) após a ovulação induzida pelo LH. Os folículos foram dissecados ou aspirados e medidos e os oócitos recuperados e submetidos à maturação, fecundação e cultivo in vitro. Os ovários do G-60 apresentaram mais oócitos viáveis (graus I, II e III) (96,6\%). A taxa de clivagem teve efeito significativo sobre o diâmetro folicular, sendo maior nos oócitos oriundos de folículos classe $3(>7 \mathrm{~mm})$. Na taxa de produção de blastocisto observou-se interação diâmetro versus fase de desenvolvimento folicular. A taxa de produção de blastocisto foi maior em oócitos obtidos de folículos com diâmetros $<5 \mathrm{~mm}$ (classe 1) no G-60 (64,5\%), de 5-7mm (classe 2) no G-96 (33,3\%) e >7mm (classe 3) no G-108 (50\%). Conclui-se que o diâmetro e a fase de desenvolvimento folicular influenciam a competência oocitária para o desenvolvimento in vitro. Nos estádios iniciais da onda folicular a produção de blastocisto foi maior em oócitos de folículos pequenos; com o avanço da onda, a produção de blastocistos foi maior em oócitos obtidos de folículos maiores.
\end{abstract}

Palavras-chave: novilha, Nelore, competência oocitária, blastocisto, divergência folicular

\begin{abstract}
The effect of follicular phase and follicle diameter on in vitro production of bovine embryos was evaluated. Follicular wave was synchronized in Nelore heifers with a progestogen for nine days and LH was administered 24 hours after progestogen withdrawal. Ovaries were recovered 60h (G-60), 96 h (G-96), or 108 h (G-108) after LH treatment. Ovarian follicles were dissected or aspirated and measured before oocytes were recovered and submitted to in vitro maturation, fertilization, and culture. The G-60 ovaries presented more viable oocyte (degrees I, II and III) (96.6\%). Cleavage rate was higher for oocytes from follicles $7 \mathrm{~mm}$ in diameter (class 3). There was a follicular phase-by-follicle diameter interaction effect on blastocyst production rate. Blastocyst production rates were higher for oocytes from follicles $5 \mathrm{~mm}$ in diameter (class 1) in the G-60 group (64.5\%), from follicles $5-7 \mathrm{~mm}$ (class 2) in the G-96 group (33.3\%), and from follicles $7 \mathrm{~mm}$ (class 3) in the G-108 group (50\%). Both the phase of follicular development and the follicle diameter influenced oocyte competence and ability for development in vitro. At the initial stages of the follicular wave, blastocyst production was higher for oocytes from small follicles. However, as the follicular wave advances, blastocyst production increases for oocytes from larger follicles.
\end{abstract}

Keywords: heifer, Nelore, oocyte competence, blastocyst, follicular deviation

Recebido em 25 de fevereiro de 2005

Aceito em 7 de fevereiro de 2007

E-mail: calie@unoeste.br.

Apoio: FAPESP 


\section{INTRODUÇÃO}

No Brasil, a produção de embriões in vitro, oriundos de oócitos colhidos de doadoras vivas por meio de aspiração folicular guiada por ultrasonografia (Ovum Pick-up, OPU), tem crescido nos últimos anos. Este procedimento permite a repetida produção de embriões de doadoras vivas de alto valor genético e contribui como uma alternativa para a superovulação, pois a OPU permite a obtenção de oócitos uma ou duas vezes por semana sem trauma aparente do trato reprodutivo, independente da fase do ciclo estral (Bols et al., 1995).

A média atual do desenvolvimento in vitro permanece em torno de $30 \%$ do total de oócitos maturados. Para 8 a 10 oócitos colhidos pela OPU há uma produção média de dois embriões transferidos em bovinos de raças européias (Galli et al., 2000). Em animais da raça Nelore (Bos indicus), Dayan et al., (2000) observaram grande variação na taxa de blastocisto entre as doadoras, com média de $42 \%$ de embriões produzidos in vitro.

A capacidade para fecundação e desenvolvimento de oócitos maturados in vivo é maior que os oócitos maturados in vitro (Blondin et al., 2002). O crescimento do oócito dentro do folículo ovariano é determinado por grande número de fatores que influenciam sua viabilidade e competência para o desenvolvimento in vitro. Esses fatores incluem: tamanho folicular (Lonergan et al., 1994; Hendriksen et al., 2000), dia do ciclo estral (Machatková et al., 1996), nível de atresia e influência do folículo dominante (Hagemann, 1999a). Quando a aspiração folicular é realizada independente da fase do ciclo estral, mais de $85 \%$ dos folículos ovarianos são atrésicos. Existem fortes evidências de que a origem do folículo é importante para determinar a qualidade do oócito (Sirard et al., 1999).

Em suma, o oócito para ser fertilizado in vivo é doado pelo folículo saudável, durante uma fase específica do ciclo estral. Enquanto, os oócitos colhidos para a produção in vitro são obtidos de folículos em diferentes etapas do desenvolvimento e em fases distintas do ciclo estral, portanto, expostos a diferentes concentrações de estradiol, progesterona, LH e FSH. Esses fatores, por sua vez, podem afetar a competência oocitária para o desenvolvimento de embriões in vitro (Wit et al., 2000).

Com o objetivo de contribuir para o aprimoramento da produção in vitro de embriões, avaliou-se o efeito do diâmetro e da fase do desenvolvimento folicular sobre a competência oocitária para a produção in vitro de embriões bovinos.

\section{MATERIAL E MÉTODOS}

Foram utilizadas 23 novilhas da raça Nelore (Bos taurus indicus), com idades variando de 28 a 40 meses, pesando em média $300 \mathrm{~kg}$, mantidas em pastagem nativa, com acesso a água e sal mineral à vontade.

Foi feita sincronização da primeira onda folicular utilizando-se implante de liberação lenta de progestógeno $^{1}$ e aplicação de $3 \mathrm{mg}$ de norgestomet e $5 \mathrm{mg}$ de valerato de estradiol no dia da inserção. O implante foi mantido por nove dias porém no oitavo dia aplicou-se $1,0 \mathrm{ml}$ de PGF2 $\alpha$ (75mg de D+Clorprostenol ${ }^{2}$ ) por via intra-vulvar. Vinte e quatro horas após a retirada dos implantes cada novilha recebeu $10 \mathrm{mg}$ de $\mathrm{LH}^{3}$ por via IM. Em seguida as novilhas foram aleatoriamente divididas em três grupos experimentais: G-60 $(n=8)$, G-96 $(n=8)$ e G-108 $(\mathrm{n}=7)$.

Os valores 60, 96 e 108 correspondem à fase do desenvolvimento folicular (em horas), da primeira onda, quando os ovários foram obtidos, no abatedouro, após a ovulação induzida pelo LH. Tendo como ponto de referência os resultados obtidos por Castilho et al. (2003), que observaram por ultra-sonografias seriadas $\mathrm{o}$ momento, em horas, da divergência no crescimento folicular em novilhas da raça Nelore, em média 84 horas após a ovulação, neste experimento avaliou-se a competência oocitária, durante a primeira onda, antes (60 horas), durante (96 horas) e após a divergência folicular (108 horas).

A ovulação não foi acompanhada para evitar estresse nos animais e comprometimento dos

\footnotetext{
${ }^{1}$ Crestar ${ }^{\circledR}$ Intervet International B.V-Boxmeer-Holanda

${ }^{2}$ Veteglan ${ }^{\circledR}$ Calier, Barcelona-Espanha

${ }^{3}$ Lutropin- $\mathrm{V}^{\circledR}$ Vetrepharm, Beleville, Ontário, Canadá
} 


\section{Castilho et al.}

resultados, dessa forma estimou-se a ocorrência da ovulação em torno de 24 a 30 horas após o LH (Castilho et al., 2000). As fêmeas foram para o abate 84, 120 e 132 horas após a aplicação do Lutropin- $\mathrm{V}^{\circledR}$. Devido ao grande número de folículos a serem dissecados, os animais do G-60 foram abatidos em um dia, e os de G-96 e G-108, no dia seguinte. Os ovários foram depositados aos pares, por animal, em sacos plásticos identificados, contendo solução fisiológica aquecida $\left(35^{\circ} \mathrm{C}\right)$ e imediatamente enviados para o laboratório; o abate não excedeu de uma hora.

No laboratório, os ovários de cinco animais de cada grupo foram dissecados; os folículos, contados e medidos com paquímetro, foram divididos pelo diâmetro em três classes de diâmetro folicular: classe $1(<5 \mathrm{~mm})$, classe $2(5$ $7 \mathrm{~mm})$, classe $3(>7 \mathrm{~mm})$. A seguir, os ovários de três novilhas nos três grupos foram aspirados com agulha $20 \mathrm{G}$.

Os oócitos, recuperados por dissecação e aspiração, foram classificados de acordo com a qualidade proposta por Lonergan (1992) em grau I, II, III - expandido, desnudo e atrésico. Em seguida foram lavados três vezes em meio TCM199 Hepes, suplementado com $10 \%$ de SFB e $50 \mu \mathrm{g}$ de amicacina, e colocados para maturar in vitro. Aproximadamente 20 oócitos foram depositados em gotas de $100 \mu \mathrm{l}$ de meio de maturação contendo TCM 199 bicarbonato suplementado com 10\% SFB (soro fetal bovino), 50UI de $\mathrm{hCG} / \mathrm{ml}, 0,5 \mu \mathrm{g}$ de $\mathrm{FSH} / \mathrm{ml}, 1 \mu \mathrm{g}$ de estradiol $/ \mathrm{ml}, 2,2 \mu \mathrm{g}$ de piruvato $/ \mathrm{ml}$ e $50 \mu \mathrm{g}$ de amicacina $/ \mathrm{ml}$, em placas de Petri mantidas à temperatura de $39^{\circ} \mathrm{C}$ em atmosfera gasosa de $5 \%$ de $\mathrm{CO}_{2}$. O período máximo de maturação recomendado é de 24 horas, entretanto os do G-60 foram maturados por 28 horas e os grupos G-96 e G-108 por 32 horas devido a problemas de ordem técnica no laboratório (problemas com o sêmen). Após esse período, os oócitos maturados foram lavados três vezes em meio de fecundação Tyrod modificado (TALP-FIV); 20 oócitos foram transferidos para cada gota de $100 \mu \mathrm{l}$ do meio Ayrod suplementado com $10 \mu \mathrm{g} / \mathrm{ml}$ de heparina e $160 \mu \mathrm{l}$ de solução PHE (penicilamina, hipotaurina e epinefrina).

O sêmen congelado foi separado em gradiente de Percoll 90 e $45 \%$ centrifugado a $900 \mathrm{~g}$ durante 30 minutos; o sedimento foi avaliado quanto ao volume, à concentração e à motilidade espermática. A concentração final foi ajustada para $25 \times 10^{6}$ espermatozóides vivos por mililitro, de modo que, ao adicionar $4 \mu \mathrm{l}$ do sêmen em cada microgota de $100 \mu \mathrm{l}$ com os oócitos maturados, resultasse na concentração final de $100 \times 10^{3}$ espermatozóides vivos por gota. As misturas foram incubadas a $39^{\circ} \mathrm{C}$ em atmosfera gasosa de $5 \%$ de $\mathrm{CO}_{2}$, para fecundação in vitro. Após 20 horas de incubação, os prováveis zigotos foram lavados três vezes em meio SOFaa (fluído de oviduto sintético), suplementado com aminoácido essencial e não-essencial, 2,5\% SFB e $0,5 \%$ de BSA (albumina sérica bovina) livre de ácidos graxos.

Os prováveis zigotos foram depositados em gotas contendo $100 \mu$ l do meio SOFaa suplementado e incubados a $39^{\circ} \mathrm{C}$ em atmosfera de $5 \%$ de $\mathrm{CO}_{2}$. Nos dias 3 e 5, após incubação, $50 \mu 1$ de SOFaa foi renovado em cada gota (feeding). A taxa de clivagem foi avaliada 48 horas após a fecundação e o desenvolvimento no oitavo dia após a maturação.

Todas as análises foram realizadas por meio do programa Statistix ${ }^{4}$. O teste do chi-quadrado foi utilizado para comparação da percentagem de folículos pertencentes às diferentes classes de diâmetro e da percentagem de oócitos pertencentes às diferentes classes de qualidade. Para a comparação da percentagem de embriões clivados e blastocistos entre as fases do desenvolvimento folicular utilizou-se teste $t$. Os tratamentos foram arranjados em um fatorial $3 \mathrm{x}$ 3 correspondentes a três fases da onda (G-60, G-96 e G-108) e três classes de diâmetro (classes $1,2$ e 3$)$.

\section{RESULTADOS}

O percentual de folículos classe $1(<5 \mathrm{~mm})$ foi menor $(\mathrm{P}<0,05)$ no $\mathrm{G}-108$ quando comparado ao G-60 (Tab. 1). A porcentagem de folículos classe $2(5-7 \mathrm{~mm})$ foi maior $(\mathrm{P}<0,05)$ em G-60 $(19,5 \%)$ e G-108 (26,1\%) (Tab. 1). Os folículos classe 3 $(>7 \mathrm{~mm})$, ausentes no G-60 surgiram no grupo G$96(14,1 \%)$ e diminuíram $(\mathrm{P}<0,05)$ no $\mathrm{G}-108$ $(4,3 \%)$ (Tab. 1).

\footnotetext{
${ }^{4}$ Analytical Software; Tallahassee, FL, USA
} 
Influência do diâmetro e da fase folicular...

Tabela 1. Folículos obtidos em diferentes fases do desenvolvimento da primeira onda nos grupos G-60, G-96 e G-108

\begin{tabular}{llll} 
& \multicolumn{1}{c}{ Classe 1 $(<5 \mathrm{~mm})$} & \multicolumn{1}{c}{ Classe 2 $(5-7 \mathrm{~mm})$} & Classe 3 $(>7 \mathrm{~mm})$ \\
\hline G-60 $(\mathrm{n}=5)$ & $80,46 \% \mathrm{a}(103 / 128)$ & $19,53 \% \mathrm{a}(25 / 128)$ & $0 \% \mathrm{a}(0 / 128)$ \\
G-96 $(\mathrm{n}=5)$ & $71,76 \% \mathrm{ab}(61 / 85)$ & $12,94 \% \mathrm{~b}(11 / 85)$ & $14,11 \% \mathrm{~b}(12 / 85)$ \\
G-108 $(\mathrm{n}=5)$ & $69,56 \% \mathrm{~b}(112 / 161)$ & $26,08 \% \mathrm{a}(42 / 161)$ & $4,34 \% \mathrm{c}(7 / 161)$ \\
\hline
\end{tabular}

Valores seguidos por letras distintas na coluna diferem entre si $(\mathrm{P}<0,05)$. G-60, G-96 e G-108= fase de desenvolvimento folicular (horas).

Não houve variação $(\mathrm{P}>0,05)$ na quantidade de oócitos graus I e III, mas a porcentagem de oócitos grau II foi maior $(\mathrm{P}<0,05)$ no $\mathrm{G}-60$ $(23,0 \%)$ (Tab. 2).

A proporção de oócitos desnudos apresentou efeito significativo $(\mathrm{P}<0,05)$ da fase folicular, sendo G-60 (2,4\%) o grupo que apresentou a menor porcentagem. No entanto, a quantidade de oócitos atrésicos não variou $(\mathrm{P}>0,05)$ (Tab. 2).

Em geral, o G-60 (96,6\%) apresentou maior número de $(\mathrm{P}<0,05)$ oócitos viáveis (Graus I, II e III) comparado aos grupos G-90 $(87,6 \%)$ e G$108(86,2 \%)$.

Tabela 2. Oócitos obtidos em diferentes fases do desenvolvimento da primeira onda nos grupos G-60, G96 e G-108

\begin{tabular}{lccccc}
\hline & \multicolumn{5}{c}{ Qualidade dos oócitos } \\
\cline { 2 - 6 } Grupos & Grau I & Grau II & Grau III & Desnudo & Atrésico \\
\hline G-60 $(\mathrm{n}=8)$ & $62,25 \% \mathrm{a}$ & $23,03 \% \mathrm{a}$ & $11,27 \% \mathrm{a}$ & $2,45 \% \mathrm{a}$ & $0,98 \% \mathrm{a}$ \\
& $(127 / 204)$ & $(47 / 204)$ & $(23 / 204)$ & $(02 / 204)$ & $(05 / 204)$ \\
G-96 $(\mathrm{n}=8)$ & $62,14 \% \mathrm{a}$ & $16,42 \% \mathrm{ab}$ & $8,57 \% \mathrm{a}$ & $7,85 \% \mathrm{~b}$ & $5,00 \% \mathrm{a}$ \\
& $(87 / 140)$ & $(23 / 140)$ & $(12 / 140)$ & $(11 / 140)$ & $(07 / 140)$ \\
G-108 (n=7) & $65,51 \% \mathrm{a}$ & $11,72 \% \mathrm{~b}$ & $8,96 \% \mathrm{a}$ & $11,72 \% \mathrm{~b}$ & $2,06 \% \mathrm{a}$ \\
& $(95 / 145)$ & $(17 / 145)$ & $(13 / 145)$ & $(17 / 145)$ & $(03 / 145)$ \\
\hline
\end{tabular}

Valores seguidos por letras distintas na coluna diferem entre si teste do chi-quadrado $(\mathrm{P}<0,05)$. G-60, G-96 e G-108=fase de desenvolvimento folicular (horas).

As taxas de clivagem e desenvolvimento até blastocisto apresentaram efeito significativo $(\mathrm{P}<0,05)$ da fase do desenvolvimento folicular, sendo a porcentagem decrescente com o avanço da onda (Tab. 3).

Tabela 3. Porcentagem de embriões clivados e blastocistos desenvolvidos de oócitos obtidos em diferentes fases do desenvolvimento folicular da primeira onda nos grupos G-60 (n=8), G-96 (n=8) e G$108(n=7)$

\begin{tabular}{lccc}
\hline & G-60 & G-96 & G-108 \\
\hline Clivagem & $80,90 \mathrm{a}(149 / 184)$ & $61,90 \% \mathrm{~b}(65 / 105)$ & $46,08 \% \mathrm{c}(53 / 115)$ \\
Blastocisto & $48,91 \% \mathrm{a}(90 / 184)$ & $22,85 \% \mathrm{~b}(24 / 105)$ & $10,43 \% \mathrm{c}(12 / 115)$
\end{tabular}

Valores seguidos por letras distintas na coluna diferem entre si teste $t \quad(\mathrm{P}<0,05)$. G-60, G-96 e $\mathrm{G}-108=$ fase de desenvolvimento folicular (horas).

Quando a taxa de clivagem foi avaliada de acordo com o diâmetro folicular, a porcentagem foi maior em folículos classe $3(>7 \mathrm{~mm})(100 \%)$ e não diferiu nas classes $1(<5 \mathrm{~mm})(56,7 \%)$ e $2(5-$ $7 \mathrm{~mm})(66,2 \%)$. Por outro lado, a taxa de blastocisto não teve efeito significativo do diâmetro folicular $(\mathrm{P}>0,05)$, porém houve interação do diâmetro pela fase do desenvolvimento folicular $(\mathrm{P}<0,05)$. Os oócitos obtidos de folículos menores que $5 \mathrm{~mm}$ (classe 1) no grupo G-60 apresentaram taxa maior de desenvolvimento (64,5\%). No G-96, a taxa mais alta de desenvolvimento foi verificada nos oócitos obtidos de folículos de $5-7 \mathrm{~mm}$ (classe 2) $(33,33 \%)$. No G-108 a maior taxa de blastocisto (50\%) foi observada em oócitos de folículos maiores que $7 \mathrm{~mm}$ (classe 3 ). 


\section{Castilho et al.}

\section{DISCUSSÃO}

Observou-se influência do diâmetro e da fase do desenvolvimento folicular sobre a competência para o desenvolvimento in vitro, sendo o início da onda o melhor momento para a obtenção dos oócitos de folículos pequenos e com o avanço da onda a competência é maior em oócitos oriundos de folículos maiores.

Após a dissecação dos folículos e separação por classe de diâmetro observou-se que a porcentagem de folículos classe $1(<5 \mathrm{~mm})$ foi mais abundante que as classes 2 e 3 , variando de $69,6 \%$ no grupo G-108 a $80,5 \%$ no G-60. Estes dados estão de acordo com os resultados obtidos em vacas Brahman em que os folículos classe 1 $(<4 \mathrm{~mm})$ foram encontrados em maior porcentagem $70 \%$ quando comparados com as classes 2 (4-8mm) (29\%) e 3 (>8mm) $(1,8 \%)$ (Fitzpatrick e Entwistle, 1997). No G-60 a quantidade de folículos classe 1 foi maior que no G-108. Essa variação da classe de diâmetro nas fases da onda foi demonstrada claramente nos estudos de dinâmica folicular por ultrasonografia (Figueiredo et al., 1997; Gambini et al., 1998; Castilho et al., 2000).

No G-60, início da onda, os folículos pequenos em maior quantidade $(80,46 \%)$, diminuiram com o avanço da onda. Os folículos classe $2(5-7 \mathrm{~mm})$ foram significativamente mais abundantes no G-108 (26,08\%). Os folículos da classe 3 $(>7 \mathrm{~mm})$ que estavam ausentes no G-60 surgiram no G-96 $(9,41 \%)$ e diminuíram no G-108 $(4,34 \%)$. Estes resultados demonstram claramente a divergência folicular (Ginther et al., 1997). A divergência folicular é definida como a maior diferença na taxa de crescimento entre o futuro dominante e maior subordinado (Ginther et al., 1996). O futuro dominante continua a crescer, é selecionado e culmina com a dominância de apenas um folículo, ao passo que o restante dos folículos recrutados no início da onda sofrem atresia. Esse evento coincide com a diminuição na concentração de FSH (Ginther et al., 2000, Castilho et al., 2003). Portanto, existe uma variação endócrina, além de substâncias produzidas pelo folículo dominante que podem afetar os folículos subordinados e conseqüentemente os oócitos. É sabido que a qualidade do oócito é de suma importância nas tecnologias de reprodução assistida (Vassena et al., 2003).
A fase da onda não influenciou a quantidade dos oócitos graus I e III, mas a porcentagem de oócitos grau II diferiu significativamente entre os tempos (G-60, G-96 e G-108), sendo no G-60 a maior proporção $(23,0 \%)$. O G-60 foi o que apresentou a maior porcentagem $(96,5 \%)$ de oócitos graus I, II e III, seguido pelo G-96 $(87,7 \%)$ e G-108 (86,1\%). Quanto maior o número de camadas de células do cumulus melhor a qualidade do oócito (Lonergan, 1992). Fukui e Sakuma, (1980), observaram que 86 a $94 \%$ dos oócitos aspirados de folículos $\leq 5 \mathrm{~mm}$ apresentavam células do cumulus, no entanto houve uma queda de aproximadamente 30\% quando os oócitos eram provenientes de folículos maiores, porém o tamanho do folículo não influenciou a maturação do oócito.

A taxa de clivagem sofreu efeito significativo $(\mathrm{P}<0,05)$ nas fases estudadas, sendo maior no G-60 (80,9\%) e menor em G-96 $(61,9 \%)$ e no G-108 (46\%). O mesmo foi observado em relação à porcentagem de blastocisto, que foi significativamente maior no G-60 (48,9\%) e menor em G-96 (22,8\%) e G-108 (10,4\%). O tempo de maturação estendeu-se por $28(\mathrm{G}-60)$ e 32 horas (G-96 e G-108), o que pode ter contribuído para a queda na taxa de desenvolvimento. Em outro estudo, no qual diferentes tempos de maturação foram testados, a porcentagem de blastocisto foi maior em oócitos maturados por 24 horas $(39,3 \%)$, mas com 28 ou 32 horas as taxas não diferiram significativamente sendo de $26,5 \%$ e $24,8 \%$, respectivamente (Ward et al., 2002). Pode-se, ainda, salientar que as taxas de clivagem e blastócisto neste experimento foram, respectivamente, $100 \%$ e $50 \%$ em oócitos classe 3 no G-108, portanto o tempo de maturação não foi decisivo para a queda na porcentagem de desenvolvimento com o avanço da onda.

Rhodes et al. (1997) observaram que a porcentagem de clivagem também foi maior em oócitos aspirados de folículos nos dias 0,1 e 2 $(54,3 \%)$ e dias 3 e $4(60,6 \%)$ da onda folicular quando comparado aos dias 5 e $6(18,2 \%)$. O desenvolvimento embrionário foi maior em oócitos aspirados de folículos durante a fase de crescimento folicular que durante fase de alta dominância (Hagemann, 1999a; Machatková et al., 2000). 
A taxa de clivagem teve efeito significativo do diâmetro folicular, sendo melhor nos oócitos oriundos de folículos classe $3(>7 \mathrm{~mm})$. Alguns trabalhos têm demonstrado que oócitos provenientes de folículos maiores se desenvolvem melhor in vitro (Loonergan et al., 1994; Hagemann et al., 1999b). No entanto, Carolan et al. (1996) não observaram influência do tamanho folicular sobre a maturação do oócito. Resultado similar foi obtido por Seneda et al. (2001), ao estudarem oócitos oriundos de folículos pequenos $(<4 \mathrm{~mm})$ e grandes $(>4 \mathrm{~mm})$ aspirados in vivo.

A taxa de blastocisto não teve efeito sobre o diâmetro, mas houve interação diâmetro versus grupo. As melhores taxas de blastocisto foram obtidas com oócitos aspirados de folículos classe $1(<5 \mathrm{~mm})$ no G-60 (64,5\%), de oócitos de folículos classe $2(5-7 \mathrm{~mm})(33,3 \%)$ no G-96 e de oócitos de folículos classe 3 (>7mm) (50\%) no G-108. Hagemann et al. (1999b) não encontraram diferença significativa na taxa de blastocisto entre oócitos obtidos de folículos 3 a 5 ou 6 a $8 \mathrm{~mm}$, mas a taxa de desenvolvimento foi significativamente maior em oócitos de folículos dominantes $(>13 \mathrm{~mm})$ durante a fase de crescimento.

Vários estudos têm demonstrado que a fase do ciclo estral e inclusive do desenvolvimento folicular influenciam a competência oocitária, uma vez que o "status" do folículo afeta diretamente o oócito. No entanto, não há consenso entre os trabalhos para definir o momento adequado da onda para se realizar a aspiração in vivo, pois os resultados variam muito.

Os oócitos obtidos de folículos maiores resultaram em melhores taxas de clivagem, por outro lado o diâmetro folicular não influenciou a taxa de blastocisto, mas houve interação diâmetro versus fase de desenvolvimento folicular, isto é, oócitos oriundos de folículos pequenos têm melhor competência no início da onda e oócitos de folículos maiores melhoram seu desenvolvimento com o avanço da onda. A fase de desenvolvimento folicular influenciou a qualidade dos oócitos e a taxa de desenvolvimento embrionário, independente do diâmetro do folículo, sendo o início da onda o melhor momento para a aspiração.

\section{AGRADECIMENTOS}

À Agropecuária J. Garcia, aos alunos Edson Ivo Turco, Karina Avelino, Andréa Renesto, Letícia Barreto, aos professores e funcionários do Departamento de Reprodução Animal - FCAVUNESP, Jaboticabal.

\section{REFERÊNCIAS BIBLIOGRÁFICAS}

BLONDIN, P.; BOUSQUET, D.; TWAGIRAMUNGO, $\mathrm{H}$. et al. Manipulation of follicular development to produce developmentally competent bovine oocytes. Biol. Reprod., v. 66, p.38-43, 2002.

BOLS, P.E.J.; VANDENHEEDE, J.M.M.; VAN SOOM, A. Transvaginal ovum-pick-up (OPU) in the cow: a new disposable needle guindance system. Theriogenology, v.43, p.677-687, 1995.

CAROLAN, C.; LONERGAN, P.; MONGET, P. et al. Effect of follicle size and quality on the ability of follicular fluid to support cytoplasmic maturation of bovine oocytes. Mol. Reprod. Dev., v. 43, p.477-483, 1996.

CASTILHO, C.; GAMBINI, A.L.G.; FERNANDES, $P$. et al. Synchronization of ovulation in crossbred dairy heifers using gonadotrophin-releasing hormone agonist, prostaglandin $\mathrm{F} 2 \alpha$ and human chorionic gonadotrophin or estradiol benzoate. Braz. J. Med. Biol. Res., v. 33, p.91-110, 2000.

CASTILHO, C.; RENESTO, A.; GARCIA, J.M. Divergência no crescimento folicular em novilhas da raça Nelore. Acta Sci. Vet., v.31, suppl., p.606-607, 2003.

DAYAN, A.; WATANABE, M.R.; WATANABE, Y.F. Fatores que interferem na produção comercial de embriões FI. Arq. Fac. Vet. UFRGS, v.28, p.181-185, 2000.

FIGUEIREDO, R.A.; BARROS, C.M.; PINHEIRO, O.L. et al. Ovarian follicular dynamics in Nelore breed (Bos indicus), Theriogenology, v.47, p.1489-1505, 1997.

FITZPATRICK, L.A.; ENTWISTLE, K.W. A comparison of dissected follicle numbers and follicle counts on the ovarian surface for the evaluation of ovarian follicular populations in Bos indicus cows. Anim. Reprod. Sci., v.46, p.179-186, 1997. 


\section{Castilho et al.}

FUKUI, Y.; SAKUMA, Y. Maturartion of bovine oocytes cultured in vitro: relation to ovarian activity, follicular size and the presence or absence of cumulus cells. Biol. Reprod., v.22, p.669-673, 1980.

GALli, C.; CROTTI, G.; NOTARI, C. et al. Embryo production by ovum pick up from live donors. Theriogenology, v.55, p.1341-1357, 2000 .

GAMBINI, A.L.G.; MOREIRA, M.B.P.; CASTILHO, C. et al. Dinâmica folicular e sincronização da ovulação em vacas da raça Gir. Rev. Bras. Reprod. Anim., v. 22, p.201-210, 1998.

GINTHER, O.J.; WILTBANK, M.C.; FRICKE, P.M. et al. Selection of the dominant follicles in cattle. Biol. Reprod., v.55, p.1187-1194, 1996.

GINTHER, O.J.; KOT, K.; KULICK, L.J. et al. Emergence and deviation of follicle during the development of follicular waves in cattle. Theriogenology, v.48, p.75-87, 1997.

GINTHER, O.J.; BERGFELD, D.R.; KULICK L.J. et al. Selection of the dominant follicles in cattle: role of two-way functional coupling between follicles-stimulating hormone and the follicles. Biol. Reprod., v.62, p.920-927, 2000.

HAGEMANN, L.J. Influence of the dominant follicle on oocytes from subordinate follicles. Theriogenology, v.51, p.449-459, 1999.

HAGEMANN, L.J.; BEAUMONT, S.E.; BERG, $M$. et al. Development during sIVP of bovine oocytes from dissected follicles: interative effects of estrous cycle stage, follicle size and atresia. Mol. Reprod. Dev., v.53, p.451-458, 1999.

HENDRIKSEN， P.J.M.; VOS， P.L.A.M.; STEENWENG, W.N.M. et al. Bovine follicular development and its effect on the in vitro competence of oocytes. Theriogenology, v.53, p.11-20, 2000.

LONERGAN, P. Studies in the in vitro maturation, fertilization and cultive of bovine follicular oocytes. 1992. 157f. Thesis (PhD). National University of Ireland, Dublin.
LONERGAN, P.; MONAGHAN, P.; RIZOS, D. et al. Effect of follicle size on bovine oocyte quality and developmental competence following maturation, fertilization, and culture in vitro. Mol. Reprod. Dev., v.37, p.48-53, 1994.

MACHATKOVÁ, M.; JOKESOVÁ, E.; PETELÍKOVÁ, J. et al. Developmental competence of bovine embryo derived from oocytes collected at various stages of the estrous cycle. Theriogenology, v.45, p.801-810, 1996.

MACHATKOVÁ, M.; JOKESOVÁ, E.; HORKY, F. Utilization of the growth phase of the first follicular wave for bovine oocyte collection improves blastocyst production. Theriogenology, v.54, p.543-550, 2000.

RHODES, F.M.; PETERSON, A.J.; McMILLAN, W.H. et al. Bovine ovarian follicle and oocyte characteristics after emergence of the first follicular wave. Theriogenology, v.47, p.149, 1997.

SENEDA, M.M.; ESPER, C.R.; GARCIA, J.M. Relationship between follicle size and ultrasound-guided transvaginal oocyte recovery. Anim. Reprod. Sci., v.67, p.37-43, 2001.

SIRARD, M.A.; PICARD, L., DERY, M. et al. The time between FSH administration and ovarian aspiration influences the development of cattle oocytes. Theriogenology, v.51, p. 699-708, 1999.

VASSENA, R.; ADAMS, G.P.; MAPLETOFT, R.J. et al. Ultrassound image characteristics of ovarian follicles in relation to oocyte competence and follicular status in cattle. Anim. Reprod. Sci., v.76, p.25-41, 2003.

WARD, F.; ENRIGHT, B., RIZOS, D. et al. Optimization in bovine embryo production: effect of duration of maturation, length of gamete co-incubation, sperm concentration and sire. Theriogenology, v.57, p.2105-2117, 2002.

WIT, A.A.C.; WURTH, Y.A.; KRUIP, A.M. Effect of ovarian and follicle quality on morphology and developmental capacity of the bovine cumulus-oocyte complex. J. Anim. Sci., v.78, p.1277-83, 2000. 